\title{
A lightning detection system for studying transient phenomena in cosmic ray observatories
}

\author{
P. Salgado-Meza, ${ }^{a}$ L. Flórez-Villegas, ${ }^{a}$ J. Peña-Rodríguez ${ }^{b}$ and L. A. Núñez ${ }^{b}$ \\ ${ }^{a}$ Universidad Industrial de Santander, Escuela de Ingeniería Eléctrica, Electrónica y de \\ Telecomunicaciones \\ Calle 9 Carrera 27, Bucaramanga, Colombia \\ ${ }^{b}$ Universidad Industrial de Santander, Escuela de Física \\ Calle 9 Carrera 27, Bucaramanga, Colombia \\ E-mail: jesus.pena@correo.uis.edu.co
}

Transients of the atmospheric electric field could cause anomalous events in the surface particle detectors of cosmic ray observatories. A better understanding of these incidents requires more accurate lightning data at the observation sites. We present the design and implementation of a monitoring system capable of detecting and storing lightning discharges. The acquisition window per event contains information of the first return stroke and the subsequent ones during 1.2 seconds at a sampling frequency of $100 \mathrm{kHz}$. The acquisition timing resolution (10 ns) allows a lightning strike location error $<10 \mathrm{~m}$. The station also records environmental data, containing temperature, barometric pressure, relative humidity, and steady-state atmospheric electric field. A GPS receiver provides the absolute time of the station. 3-dimensional mapping of lightning discharge can be reconstructed in detail using a monitoring network of at least three stations. We present preliminary measurements during a thunderstorm episode (2019-11-09). The event lasts about 2 hours, recording a maximum electric field peak of $\sim-15 \mathrm{kV} / \mathrm{m}$. The atmospheric potential was $\sim 27 \mathrm{MV}$ with an estimated thunderstorm cloud-base height of $\sim 2 \mathrm{~km}$. At least four lightning events occurred during the thunderstorm period. Such discharges released an electric field $>5 \mathrm{kV} / \mathrm{m}$.

$37^{\text {th }}$ International Cosmic Ray Conference (ICRC 2021)

July 12th - 23rd, 2021

Online - Berlin, Germany 


\section{Introduction}

Cosmic rays (CRs) are particles reaching the Earth after crossing interstellar space. High energy CRs originate outside the solar system, and most of the low-energy $(<10 \mathrm{GeV})$ particles have a solar origin. When CRs enter the terrestrial atmosphere, they interact with the atoms generating Extensive Air Showers (EAS) of secondary particles [1].

Secondary particles, mainly electrons/positrons, can be affected by the atmospheric electric field. They can be accelerated [2] causing Relativistic Runaway Electron Avalanches [3] increasing the secondary flux at ground level. The atmospheric electric field can rise to hundreds of $100 \mathrm{kV} / \mathrm{m}$ during thunderstorm episodes. The minimum electric field for triggering the avalanche is roughly $286 \mathrm{kV} / \mathrm{m} \mathrm{[4,5].} \mathrm{Some} \mathrm{investigations} \mathrm{demonstrate} \mathrm{that} \mathrm{the} \mathrm{flux} \mathrm{of} \mathrm{heavier} \mathrm{particles} \mathrm{such} \mathrm{as} \mathrm{muons}$ can also be modulated by the atmospheric electric field $[6,7]$.

The flux variation of charged particles depends on the polarity of the atmospheric electric field $[8,9]$. Negative charge particles are accelerated towards the ground during negative electric fields, enhancing the particle counting at the detector level. On the other hand, in a positive electric field, the counting ratio at ground level decreases [10]. These observations explain the electron/positron flux variation which CRs observatories have observed during thunderstorms.

The lack of electric field monitoring networks inside CR observatories limits understanding the underlying phenomena responsible for the recorded anomalous events [11, 12]. The data crosschecking between the particle counting rate and the transitory electric field shall help to clarify such phenomena.

We present preliminary results of the development of a modular monitoring station capable to measure atmospheric electric field variations during thunderstorm episodes. The station allows us to create a temporally synchronized network for finding correlations between secondary flux and electric field variations, as well as, it is able to estimate the localization of lightning strike points.

\section{Quasi-static electric field}

There exist several technologies for measuring slow variations of the atmospheric electric field. These are inductive probes, field mills, and optical sensors [13]. E-field mills are widely used to measure slowly-varying electric fields associated with cloud charging processes.

The steady electric field is shielded and unshielded by a moving grounded shutter placed over a sensing electrode in the E-field mill. The charge induced on the sensitive electrode flows to the ground when the shutter screens the external electric field and flows back to the sensing uncovered electrode. The resulting sinusoidal current is proportional to the electric field magnitude [14].

The implemented E-field sensor, composed of three Bakelite plates (shutter, sensing electrode, and ground), have a $1.4 \mathrm{~mm}$ width with a copper layer of $35 \mu \mathrm{m}$. The dielectric is made of a $2 \mathrm{~mm}$ acrylic layer, and the sensing electrode has a diameter of $62 \mathrm{~mm}$ and three blades separated by $120^{\circ}$. The shutter radius is $5 \mathrm{~mm}$ larger than the sensing plate to interrupt the encoder for generating the signal phase. The ground plate is connected to the DC motor chassis, and the estimated E-field mill capacitance is around $6 \mathrm{pF}$.

For improving the sensor sensitivity, we evaluated the induced voltage on the sensing plate and the rotation frequency of the DC motor, which is a third of the frequency of the generated signal 
due to the sensing plate shape. The signal frequency spanned from 200 to $600 \mathrm{~Hz}$, observing output voltages from 8 to $14 \mathrm{Vpp}$. Considering the power consumption, vibrations, and acoustic noise, we selected a signal frequency of $260 \mathrm{~Hz}$, meaning a motor rotation frequency of $87 \mathrm{~Hz}$.

\subsection{Signal conditioning}

The sensor prototype front-end comprises a resistive measuring range selector, an op-amp follower for impedance coupling, an input DC decoupler, and an op-amp adder for the signal baseline setting.

One of the advantages of mill sensors is determining the induced field direction by comparing the rotation phase of the shutter and the phase of the sensing plate signal using a precision rectifier. We used the Mixer component of the Programmable System on Chip (PSoC 5LP from Cypress Semiconductor) for carrying out such operation. The rectification task requires inputting the E-field mill signal, the phase digital signal generated by an encoder (EE-SJ3), and the reference voltage, which indicates the DC level over which the comparison occurs.

The Mixer inverts the sinusoidal signal when the phase is 0 , and a low-pass filter smoothes the signal ripple. The resulting modulated signal has a RMS value proportional to the magnitude of the induced electric field. We used a $1 \mathrm{mF}$ capacitor connected in parallel to the output with a frequency cut of $\sim 0.2 \mathrm{~Hz}$.

\subsection{Signal digitization and transmission}

A 20-bit delta-sigma ADC performs the signal digitalization. The ADC integrated into the PSoC 5LP has a single-ended configuration, a sampling frequency of 183 SPS (Samples Per Second), and an input range from 0 to $5 \mathrm{~V}$. One ADC unit is equivalent to $4.5 \mu \mathrm{V}$.

The digitized E-field data is transmitted by $\mathrm{I}^{2} \mathrm{C}$ protocol with a speed of $100 \mathrm{kbps}$. A $C$ script controlled the $\mathrm{I}^{2} \mathrm{C}$ module. The code configures the sensor initialization, device address (0x60) and memory sub-addresses. Since the communication protocol sends 8-bit data packages, the ADC data (20 bits) is split into four 8-bit-blocks stored in the device sub-addresses from $0 \mathrm{x} 00$ to $0 \times 03$.

\subsection{Calibration}

The E-field sensor calibration was performed using a controlled E-field generator following the methodology proposed by Cui et al. [15]. The field generator was a squared capacitor made of two parallel plates $20 \mathrm{~cm}$ side and $10 \mathrm{~cm}$ separation. A DC/DC EMCO C20 power source provided the high voltage applied between the generator plates. The generator controlled by a Raspberry Pi board employs a 12 bits DAC MCP4725 generating a E-field range of $0-20 \mathrm{kV} / \mathrm{m}$ with a resolution $\sim 5 \mathrm{~V} / \mathrm{m}$. The sensor was located inside the parallel capacitor under E-fields ranging from 0 to $2 \mathrm{kV} / \mathrm{m}$ with $\sim 400 \mathrm{~V} / \mathrm{m}$ steps. The sensor maximum measuring range is $0-500 \mathrm{kV} / \mathrm{m}$.

\section{Lightning}

Charge accumulation inside thunderstorm clouds can break down the resistance of air unchaining fast variations ( $1 \mathrm{kHz}-1 \mathrm{MHz})$ of the atmospheric electric field: the most common is lightning. A parallel plate antenna composes the lightning detection system front-end. The device detects 
the E-field variation during lightning discharges. An active integrator converts the antenna current in a voltage proportional to the induced E-field. A fast ADC digitizes the voltage signal, and a NOR Flash memory temporally stores it. A voltage-over-threshold trigger determines the event truthfulness starting the information transmission towards the single computer board (Raspberry Pi 2).

\subsection{Antenna}

The parallel-plate antenna consists of two circular aluminium plates $40 \mathrm{~cm}$ diameter separated by $2 \mathrm{~cm} \mathrm{[16].} \mathrm{The} \mathrm{top} \mathrm{plate} \mathrm{senses} \mathrm{the} \mathrm{electric} \mathrm{field,} \mathrm{while} \mathrm{the} \mathrm{lower} \mathrm{one} \mathrm{is} \mathrm{grounded.} \mathrm{The} \mathrm{antenna}$ transmits the signal using an RG51 coaxial cable with a BNC connector end.

The antenna current due to the induced electric field $E$ is

$$
I=\epsilon A \frac{d E}{d t}
$$

where $A$ is the aluminum plate area and $\epsilon$ is the dielectric material permittivity.

\subsection{Integrator}

We can deduce from Eq. 1 that the integration of the antenna signal gives us an estimation of the induced electric field as follows,

$$
V=\frac{1}{C} \int I d t=\frac{\epsilon A E}{C}
$$

where $C$ is the antenna capacitance. The measured electric field is

$$
E=\frac{C V}{\epsilon A}
$$

We implemented an active integrator on an OPA4228 operational amplifier. The op-amp has a $120 \mathrm{~dB}$ CMRR and a bandwidth of $33 \mathrm{MHz}$. An adder cascades the integrator to invert the signal and increase the baseline. The circuit's frequency response was simulated en PSpice and tested by varying the input frequency from $100 \mathrm{~Hz}$ to $20 \mathrm{MHz}$. The simulated frequency cut was $\sim 10.6 \mathrm{MHz}$ and the measured $\sim 4 \mathrm{MHz}(-3 \mathrm{~dB})$ [16]. The circuit noise presents a normal distribution centred in $0 \mathrm{mV}$ and with a standard deviation $\sim 3.49 \mathrm{mV}$.

\subsubsection{Lightning spectral analysis}

Fig. 1 shows the 02032020 (March 2, 2020) lightning event recorded at Bucaramanga, Colombia. A Tektronix TDS 2002B (1 GS/s) establishes the sampling frequency of the digitisation stage in the analysis of the lightning signal frequency spectrum. The signal presents a first return stroke of $\sim 4 \mathrm{~V}(200 \mathrm{~V} / \mathrm{m}$ above the antenna place) with subsequent discharges at $\Delta t \sim 0.1 \mathrm{~s}, \sim 0.25 \mathrm{~s}, \sim 0.35 \mathrm{~s}$, and $\sim 0.39 \mathrm{~s}$. The recording window was set to $0.7 \mathrm{~s}$.

We characterised the noise (red) and signal (green) spectrum by applying the FFT in two portions of the recorded signal. The principal frequency components of the lightning signal are below $\sim 4 \mathrm{kHz}$, while noise components extend to $\sim 20 \mathrm{kHz}$. 

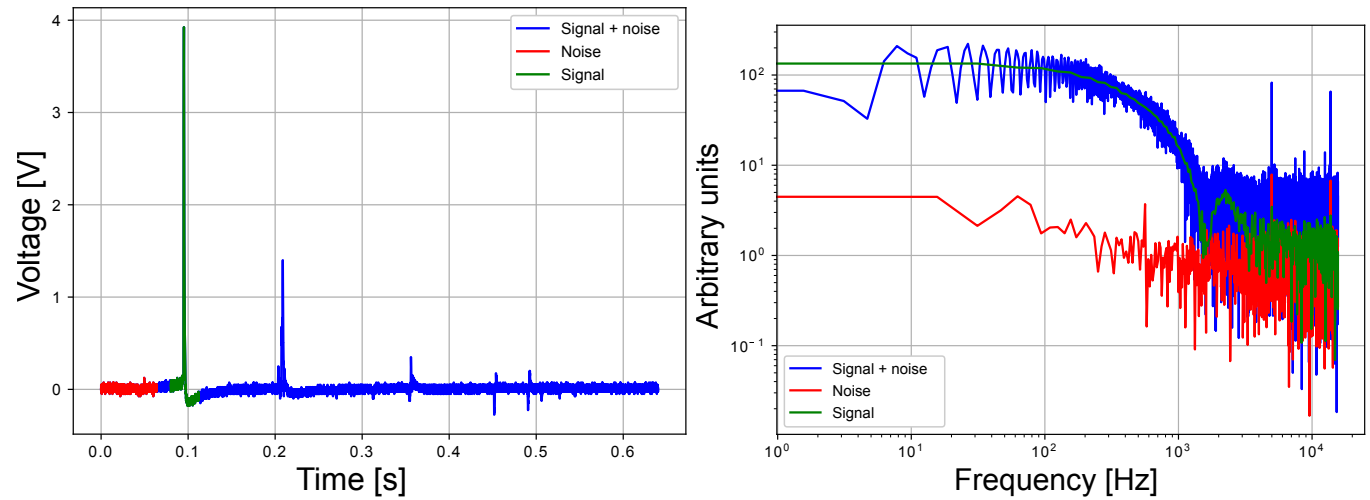

Figure 1: Lightning signal recorded on March 2, 2020, at Bucaramanga-Colombia. The signal was split into noise (red-line) and discharge (green-line) portions to analyze their frequency spectrum. b) Fast Fourier Transform of the recorded signal. The frequency components of the discharge (green-line) are below $\sim 4 \mathrm{kHz}$ and the noise (red-line) extend to $\sim 20 \mathrm{kHz}$.

\subsection{Digitization and storage}

A Spartan 6 FPGA Development Board (Mimas) manages the acquisition process. The FPGA generates the $1 \mathrm{MHz}$ ADC clock, applies the voltage-over-threshold trigger, addresses the signal data to 16-Mbit flash memory (SST39VF1602C ${ }^{1}$ ), and transmits the data to the Raspberry Pi.

When the lightning event exceeds the detection threshold $(\sim 500 \mathrm{mV})$, the data acquisition system records a $1.2 \mathrm{~s}$ window at $100 \mathrm{kHz}$. Each recorded event has an associated timestamp with $10 \mathrm{~ns}$ resolution and GPS synchronization (UTC). We tested the lightning detection hardware by injecting square pulses emulating electrical discharges (50 ms width and $1 \mathrm{~V}$ height) using a Tektronix AFG1022 signal generator. This test-bench allowed us to evaluate acquisition timing.

\subsection{Monitoring station}

The monitoring station includes the lightning detection system, the atmospheric electric field mill, and environmental sensors. A single computer board (Raspberry Pi) operates the station running under Raspbian Wheezy. The BME280² sensor measures temperature, relative humidity, and atmospheric pressure with an accuracy of $\pm 1{ }^{\circ} \mathrm{C}, \pm 3 \% \mathrm{RH}$, and $\pm 1 \mathrm{hPa}$ respectively.

A Venus 638FLPx chip performs GPS positioning with a spatial accuracy of $2.5 \mathrm{~m}$ and a time accuracy of $60 \mathrm{~ns}$, communicating via UART protocol at 9600 bauds and needs a $3.3 \mathrm{~V}$ powering. The positioning data contains UTC, latitude, N/S indicator, longitude, E/W indicator, and altitude. The time synchronization of the acquisition system depends on the PPS signal. The estimation of the lightning strike point is obtained by using a trilateration algorithm based on the GPS data [17].

The four layer (bottom-up) hardware are the Raspberry Pi 2, the environmental layer (PSOC 5LP, GPS Venus 638FLPx, and BME280), the Spartan 6 FPGA Development Board, and the lightning detection system. Figure 2 displays the station hardware.

1https://www . microchip.com/wwwproducts/en/SST39VF1602C

${ }^{2}$ https: //www . bosch-sensortec. com/products/environmental-sensors/humidity-sensors-bme280/ 


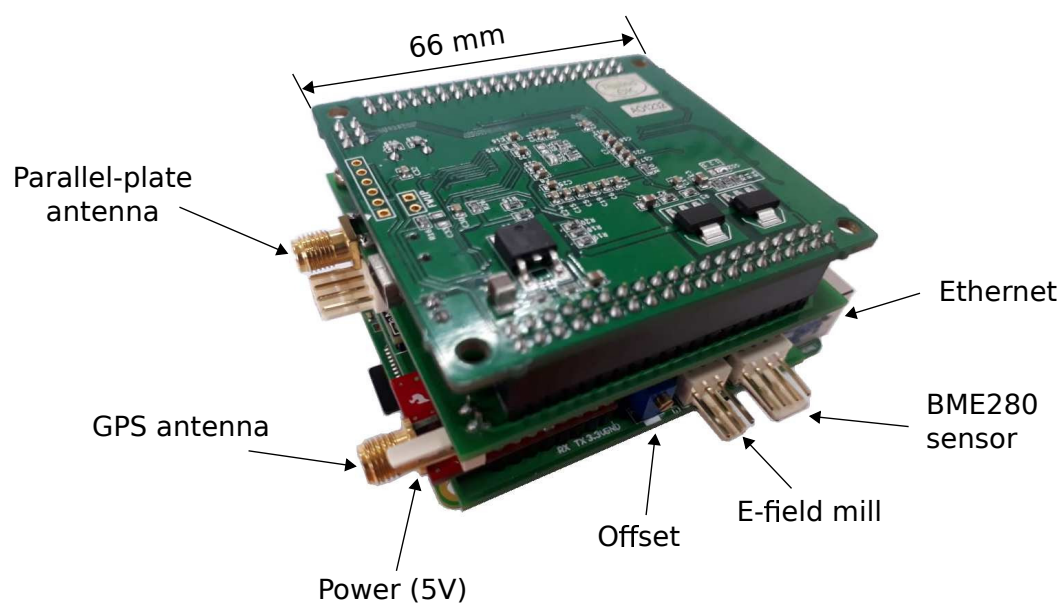

Figure 2: Monitoring station hardware. The lightning and environmental readout electronics stack on the Raspberry Pi single computer board as removable shields. External connectors for powering, weather sensors, GPS antenna, parallel plate antenna, E-field mill, and Ethernet encircle the station hardware.

\subsection{First measurements}

We report preliminary measurements recorded during a thunderstorm event on 2019-11-09 at Bucaramanga-Colombia. The event lasts about 2 hours, recording a maximum electric field peak of $\sim-15 \mathrm{kV} / \mathrm{m}$. The atmospheric electric field recovers the steady-state after at least half-hour of continuous lightning activity, showing a ripple $\sim 4 \mathrm{kV} / \mathrm{m}$.

The thunderstorm cloud-base height $(\sim 2 \mathrm{~km})$ was estimated by using a model based on the relative humidity and temperature [18]. The cloud-to-ground potential is proportional to the measured electric field and inverse to the cloud base distance. The atmospheric potential was $\sim 27 \mathrm{MV}$ at the electric field maximum. The environmental temperature dropped $3.5^{\circ}$ during the thunderstorm beginning. The relative humidity decreased from $88 \%$ to $76 \%$ due to a wind speed increment (dissipation of the water molecules).

At least four lightning events occurred during the thunderstorm period, releasing electric fields above $5 \mathrm{kV} / \mathrm{m}$. The first event represented an electric field variation of close $8.5 \mathrm{kV} / \mathrm{m}$ in less than one minute. The thundercloud charge accumulation was released during $(\sim 10 \mathrm{~min})$ by lightning discharges until the electric field decreased around the baseline. The cloud-to-ground current estimation shall be possible if we know the lightning strike distance.

\section{Conclusions}

Several studies have demonstrated a direct relationship between the atmospheric electric field and the flux of charged particles at ground level. In this note, we presented a monitoring station design and first measurements of the atmospheric electric field, lightning, and environmental variables (temperature and relative humidity) involved in thunderstorm episodes. The station acquisition system fulfils the timing and triggering requirements needed to cross the recorded data with the counting rate measurements of particle detectors. 

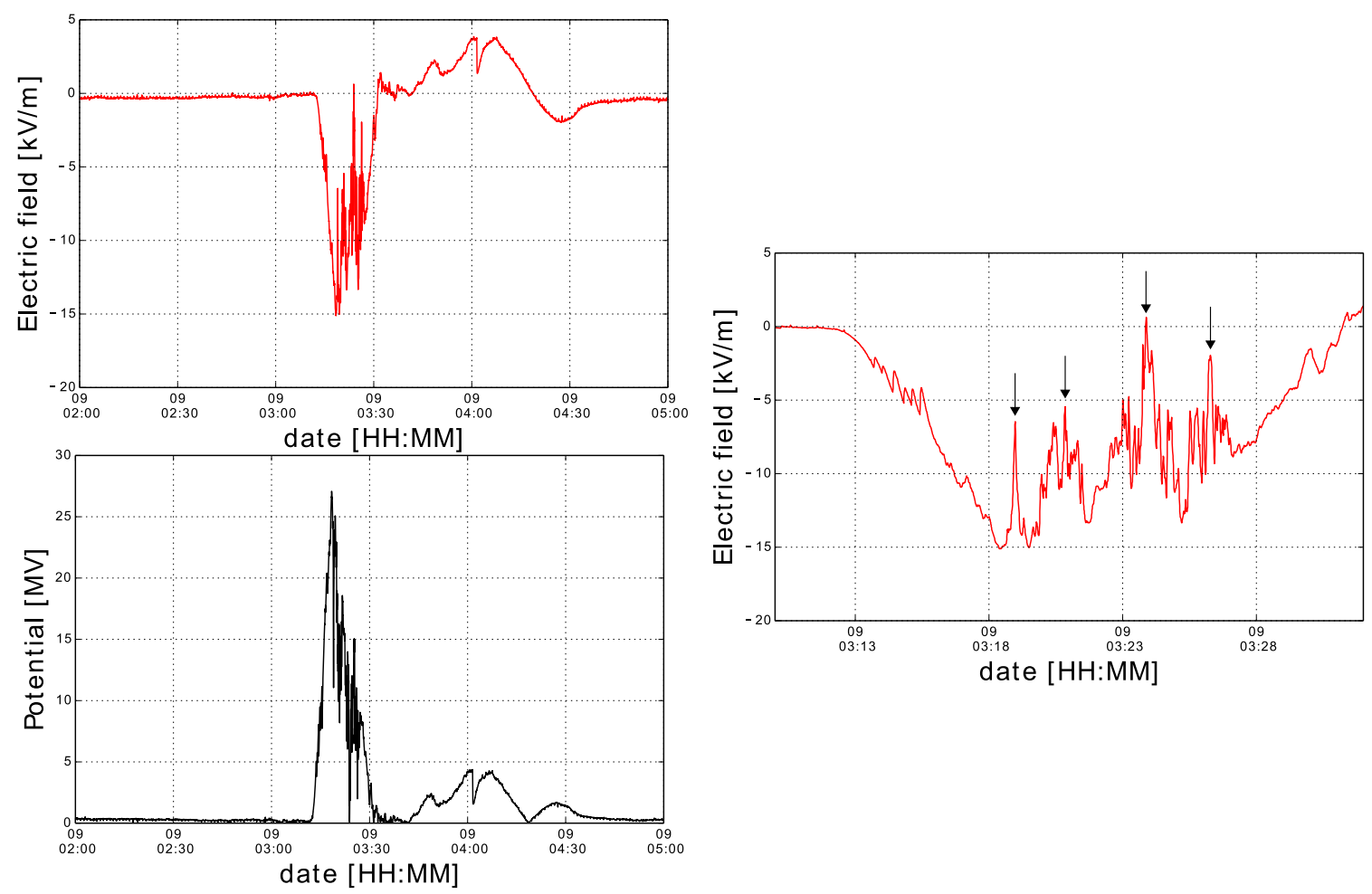

Figure 3: Results of the atmospheric data taking during the 2019-11-09 thunderstorm episode. A negative electric field (red-line) with a maximum of $-15 \mathrm{kV} / \mathrm{m}$ was recorded after the thundercloud formation $\sim 2 \mathrm{~km}$ above the observation place. The absolute value of the cloud-ground potential (black-line) was estimated using the atmospheric electric field and the cloud base. The cloud base was modeled by using measurements of temperature and relative humidity. Lightning events are indicated by black-arrows.

Preliminary measurements showed a correlation between the environmental temperature and relative humidity during the 2019-11-09 thunderstorm development. The atmospheric electric field rose to $-15 \mathrm{kV} / \mathrm{m}$ with a cloud-ground potential $\sim 27 \mathrm{MV}$. Additionally, we identified some lightning events which caused an electric field variation of the order of $8.5 \mathrm{kV} / \mathrm{m}$.

\section{References}

[1] M. Spurio, Particles and Astrophysics, Springer International Publishing (2015), 10.1007/978-3-319-08051-2.

[2] T.C. Marshall et al., Observed electric fields associated with lightning initiation, Geophysical Research Letters 32 (2005) .

[3] L.P. Dwyer, Low-energy electron production by relativistic runaway electron avalanches in air, Journal of Geophysical Research: Space Physics 116 (2011) .

[4] A.B. Skeltved et al., Constraints to do realistic modeling of the electric field ahead of the tip of a lightning leader, Journal of Geophysical Research: Atmospheres 122 (2017) 8120. 
[5] R. Colalillo, TGE at UHECR energies, in Thunderstorms and Elementary Particle Acceleration (TEPA-2019), 2019.

[6] W. Jun-Fang et al., Effect of thunderstorm electric field on intensity of cosmic ray muons, Acta Physica Sinica 61 (2012) 159202.

[7] V.V. Alexeenko et al., Transient variations of secondary cosmic rays due to atmospheric electric field and evidence for pre-lightning particle acceleration, Physics Letters A 301 (2002) 299.

[8] B. Bartoli et al., Observation of the thunderstorm-related ground cosmic ray flux variations by ARGO-YBJ, Physical Review D 97 (2018).

[9] B. Zhao et al., Effects of thunderstorms electric field on intensities of positrons, electrons and photons at daocheng, in 36th International Cosmic Ray Conference (ICRC2019), vol. 36, 2019.

[10] L. Dorman, Cosmic rays in the Earth's atmosphere and underground, vol. 303, Springer Science \& Business Media (2013).

[11] R. Colalillo et al., Peculiar lightning-related events observed by the surface detector of the pierre auger observatory, in Proceedings of 35th International Cosmic Ray Conference PoS(ICRC2017), Sissa Medialab, Aug., 2017, DOI.

[12] K. Merenda et al., Atmospheric electricity studies at the pierre auger observatory: Signal comparisons between lightning and cosmic ray events, EPJ Web of Conferences 210 (2019) 05007.

[13] R. Miles, T. Bond and G. Meyer, Report on non-contact dc electric field sensors, Tech. Rep. Lawrence Livermore National Lab.(LLNL), Livermore, CA (United States) (2009).

[14] V.A. Rakov, Fundamentals of Lightning, Cambridge University Press (2016), 10.1017/cbo9781139680370.

[15] Y. Cui et al., Model, design, and testing of field mill sensors for measuring electric fields under high-voltage direct-current power lines, IEEE Transactions on Industrial Electronics $\mathbf{6 5}$ (2017) 608.

[16] P. Salgado-Meza and L. Florez-Villegas, Diseño e implementación de una estación metereológica para la medición del campo eléctrico atmoaférico en observatorios de rayos cósmicos, Master's thesis, Universidad Industrial de Santander, 2020.

[17] I. Mialdea-Flor et al., Development of a low-cost IoT system for lightning strike detection and location, Electronics 8 (2019) 1512.

[18] M.G. Lawrence, The relationship between relative humidity and the dewpoint temperature in moist air: A simple conversion and applications, Bulletin of the American Meteorological Society 86 (2005) 225. 